\title{
The Application of Whole Process Error Prevention Quality Management System in Rail Transit Signalling System
}

\author{
Fengqin Zhang ${ }^{1+}$, Lijuan Lu ${ }^{1}$, Dongmei Xue ${ }^{1}$ \\ Quality Management Department, Zhejiang United Science and Technology Co., Ltd, Hangzhou, China
}

\begin{abstract}
With the rapid development of urban rail transit in China, the signalling system, as an important part of it, plays a vital role in safety, punctuality, efficient operation. The traditional management model only attaches importance to product development and design based on error correction and local prevention, which has been unable to meet today's requirements. This paper presents error prevention quality management system framework of the whole life cycle of signalling system and its application.
\end{abstract}

Keywords: error prevention, quality management, reliability, safety, signalling system

\section{Introduction}

Railway signalling system is the core component of traffic railway. Due to its high complexity, high safety and high reliability, the traditional management mode based on error correction and local prevention, is achieved by "finding" non-conformance, conducting cause analysis, developing corrective and preventive action, improving design and verifying execution effect. This management model lacks quality prevention and control of the whole process including the planning, design, technology, manufacturing, verification and validation with high cost. Signalling system quality management system requires maximum control of the occurrence of errors to ensure the safety and reliability of the system. In this context, in order to promote the quality management of rail transit signalling system to enter a more mature stage, it shall establish whole process error prevention quality management system in rail transit signalling system, including bidding management, design and development, technology process control, manufacturing, verification and validation and delivery, etc. thus ensuring the safety and reliability of the signalling system.

\section{The Framework of Error Prevention Working System of the Whole Development Process of Signalling System Product}

With the guidance of quality policy of the company, the PDCA management method has been followed, to continuously sum up the experience of design development and production, deeply communicate with customers, so as to develop error prevention management mechanism with the goal of doing things right for the first time. Through top to bottom promotion by decision-making level, management level and implementation level, and according to customer demands and opinions, working system of the whole process shall be continuously optimized and improved so as to form Closed-loop management. In the quality management system, it requires to strengthen the process control method of product realization, form technical foundation such as the design and development, process technology, manufacturing, verification and validation, etc. and conduct comprehensive quantitative management through the standard specifications, quality requirements, statistical techniques and tools and error prevention application. The framework of error prevention quality management system of the whole process of signalling system product is shown in Figure 1.

\footnotetext{
Corresponding author. Tel.: + 8618905816600

E-mail address: zhangfengqin@unitedmne.com.
} 


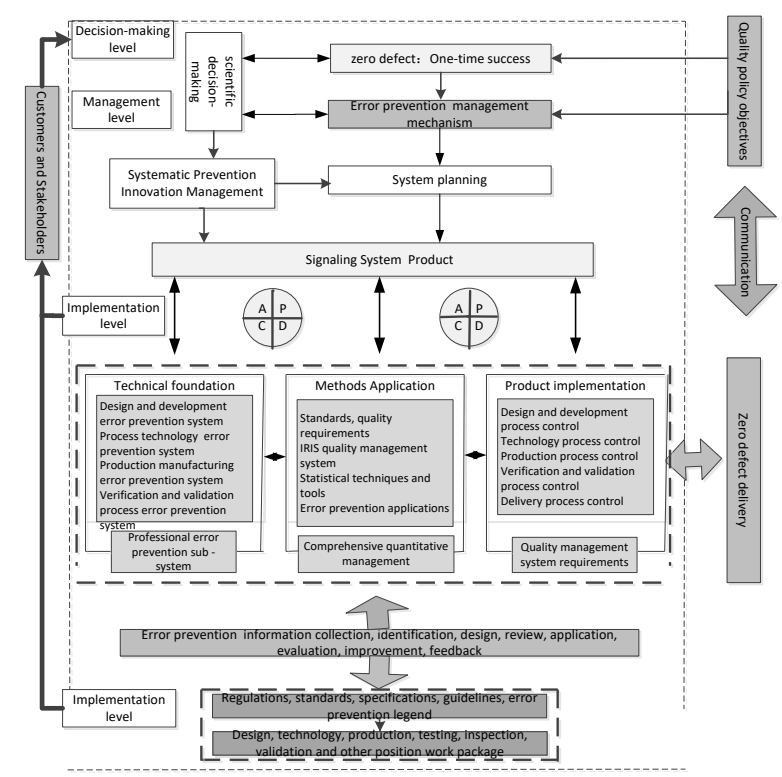

Fig. 1: The framework of error prevention quality management system of the whole process of signalling system product

\section{Error Prevention Working Subsystem of the Whole Process of Signalling System}

Error prevention working system is divided into six subsystems in accordance with the life cycle model of the development of signalling system product, which are design development process, process technology, production process, verification and validation respectively. Each subsystem is mutually related and integrated and support each other. In each sub-system, through the summarization of practical experience and lessons of history of all links of the whole development process, specification documents of all links are compiled, and weak links and possible quality risks of the whole development process are identified to establish human error prevention subsystem. Error prevention subsystem of the whole development process effectively implants key points of error prevention into key links of development, to ensure that human errors can be avoided in all aspects of the links, including design, production and delivery.

\subsection{Error prevention working subsystem of design and development process}

In the phase of bidding management, the requirement analyst shall check the tender document one by one, after assessment and discussion, correctly communicate to each relevant personnel to ensure the correct understanding and commitment. Before the submission of tender, the requirements from customers and laws and regulations are understood by the method of multiple authentication.

In the phase of proposal certification and preliminary design, the designer shall complete relevant proposal certification in accordance with proposal certification guidance and preliminary design guidance. Safety engineers shall carry out preliminary hazard analysis. In order to improve the product quality from the source, standardize the design process and achieve the "zero defect" for the whole life cycle of product, according to the requirements of error prevention working subsystem of design process, in addition to the position specification document, dozens of guidance documents are developed in design phase, such as Preventive and Corrective Measures, Hardware Development Specification, Software Design and Development Specification, Product Release Instruction, etc.

Hardware design and implementation play a key role in the safety and quality of signalling system. It is necessary to analyze and verify hardware requirements one by one in the system architecture through the requirement traceability tool, and perform SHA hazard analysis on the hardware requirements that have been verified, and complete relevant analysis on schematic diagram and BOM by hardware FMECA analysis, single fault mode analysis, multiple fault mode analysis, Fault Tree Analysis (FTA), reliability allocation, etc. In the phase of the drawings and design, the designer shall design and compile FTA report, technology 
summarization report and error prevention measure application report in accordance with technology document and error prevention document, and strictly implement review procedure.

Whether software development process is in accordance with EN50128 standard requirements, it will exert a direct impact on the quality of software product, therefore, it is necessary to establish software development error prevention sub-system, and control all links. In addition to the development of Software Design and Development Specification, when the software requirements are analyzed, it is required by the subsystem to decompose the requirements one by one according to software requirements. Requirements are traced by the Reqtify tool to ensure that all of the requirements can be effectively implemented. The correctness of the software requirements are verified by software FMEA analysis. The key quality assurance measures of the software include code review, static analysis, unit testing, software integration testing and validation testing. All tests are in strict accordance with the standards EN50128, and test defects are tracked and solved in accordance with Defect Management Regulation, till it is closed. When the software is changed, it shall strictly follow Change Control Specification to describe the change causes and implementation solution in detail. Safety engineers conduct RAMS analysis on the modified contents to ensure software quality control.

\subsection{Error prevention working subsystem of technical process}

Process technology error prevention measures require the technologists to take into account the potential problems and risks in the manufacturing process in the phase of technology document preparation and take relevant measures to avoid, which include technology FMEA analysis, technology document review, technology quality problems drawing inferences, visualization technology, etc. Technology review includes critical technology review, technology general plan review, new technology and new technology application review. The technologists carry out the work through the standardization guidance document to prevent human error in the process. In the process of technology procedure development, process parameters and product standard value in the operation instruction shall be examined to check whether the contents conform to the latest version of the technical standards, and can provide an accurate basis for production. Visual management is achieved through the test tooling, timely feedback quality, fault information and other aspects, establish early warning notification mechanism, understand the existing problems, find the reasons by analysis for rectification control and problem prevention.

\subsection{Error prevention working subsystem of production and manufacturing process}

Production and manufacturing process error prevention plays an important role in preventing product defects and improving product pass rate. In the production process, the execution time sequence shall be taken into consideration to avoid cross-operation, emphasizing "self-inspection, mutual inspection, special inspection" and other control measures. The operators are required to operate in strict accordance with the technology requirements in the product manufacturing process, at the same time the error prevention working subsystem of production and manufacturing process requires the technologists to accumulate the history of out-of-tolerance in the manufacturing process, summarize and refine in the technology documents in order to improve product specification and consistency, and reduce the occurrence of human error. In the process of product assembly and debugging, error prevention working subsystem requires that the index be quantified, by marking and emphasizing error prone links in technology documents, it can effectively ensure the operator can notice the weak link of human error, so as to avoid the assembly step error and assembly failure. Meanwhile, the operators are warned by means of visual kanban management, which can effectively avoid the subsequent machine process human error, thereby effectively improving the assembly one-time pass rate.

\subsection{Error prevention working subsystem of verification and validation manufacturing process}

The verification and validation process runs through the whole process of product realization. The human errors of verifiers and validators will not occur by verification and validation error prevention working system. The product monitoring and measurement points and mandatory inspection points are analyzed and identified by the methods of PHA, SHA, FMEA, FTA from the design level, technology level 
and manufacturing level, furthermore, these inspection points are defined through the checklist and guidance and the exit criteria for each phase is defined. In the whole life cycle of the product, verification is performed by means of the relevant checklists according to the exit criteria for each phase and the verification report is output. At the same time, the verification process shall follow the appropriate guidance documents such as Identification and Traceability Management Guidance, Product Monitoring and Measurement Procedure, Nonconforming Product Control Procedure, etc. The verification shall ensure the integrity of the records of the whole process. In order to ensure that the system requirements meet the customer's final requirements, the validator is required on the basis of verification. Validator is completely independent from the development and design staff. The requirements and traceability shall be reviewed to ensure all of these are fulfilled. The correctness, consistency and adequacy of test cases and executed tests shall be checked. The related hazard logs shall be reviewed to ensure all hazards are closed or transferred. For unclosed issues, impact analysis shall be conducted and finally form the system validation report.

\section{PDCA Management Method}

The whole process error prevention management system has always followed the PDCA management approach to effectively guide the planning, implementation, effect inspection, measure consolidation and continuous improvement of the whole process error prevention management system. P, D, C, A respectively represent four stages of the system promotion in the whole process error prevention working system, as shown in Figure 2.



Fig. 2: PDCA management method

By following the scientific PDCA management method, and carrying out PDCA reciprocating cycle continuously in the quality management system, error prevention working system of the whole development process of signalling system has been established and perfected, and the error prevention technology documents and position error prevention key points has been detailed, and error prevention working requirements and implementation methods of quality management system has been continuously supplemented and improved. 


\section{Application}

The establishment of error prevention working system of the whole development process for rail transit signalling system effectively controls and prevents human error of the whole development process for rail transit signalling system. The effect of human error prevention of all links is obvious and the product onetime passing rate is in great increase. In the design phase, the correctness and effectiveness of the design technical documents and design changes are guaranteed by means of design review and three-level approval, to prevent the occurrence of human design errors. The designers identify the key features of products through product requirements analysis, single failure mode analysis, reliability, safety and zero quality defect of historical product and identify the weakness of product. On the basis of comprehensive understanding of the key characteristics of the product design and comprehensive summarization of the production process, the technologists summarize the links and factors that affect the stability and reliability of the product quality, transform it into controllable and measurable process key features, and focus on control and detection of these error prevention points in all links of production and debugging. In the production phase, processing error prevention points are arranged to prevent unreasonable processing methods and processes. In the testing phase, simulation testing tools are applied to comprehensively simulate signalling system application environment, comprehensively verify the safety and reliability of the system and avoid errors caused by human factors in the testing process by automated testing tools. Simulation test tool records are well documented which are available at any moment and it can effectively prevent human error. Multimedia records of the components, assembly and the overall products are documented as the basis for product acceptance, so as to improve product quality traceability, conveniently and timely summary the root causes of non-conforming products, constantly fill product quality problem library, and effectively improve error prevention system.

\section{Conclusion}

Error prevention management system implements the "zero defect" management philosophy, continues to expand and deepen the meaning of error prevention, employs tools and methods of error prevention which is not limited to the traditional seven new and old tools, SPC process control technology, FMEA fault mode analysis. While adhering to the quality management methods of rail transit, effective process control methods and measures such as quality and reliability engineering technique, quantitative control technique, visual management technique and zero-defect, etc. are innovatively applied in error prevention management system and play an important role in practice.

\section{Acknowledgements}

This work is supported by the funding from the National Science and Technology Infrastructure Program of China under Grant 2015BAG19B03

\section{References}

[1] Qiang Xue, Signalling System Deployment Design Process Quality Control[J]. Railway Signalling \& Communication Engineering, 2015, 12(5): 93-96.

[2] Jinyuan Zhao, Yuanguan She, Xiaojun Gu, Research and Application of Tracking and Management System of Total Process Quality in Tanggang[J]. Metallurgical Industry Automation, 2013, 37(1): 16-21

[3] GB/T19001-2016 Quality Management Systems—-Requirements

[4] EN50126 Railway Applications - The Specification and Demonstration of Reliability, Availability, Maintainability and Safety (RAMS), 1999

[5] EN50128 Railway Applications - Communication, Signalling and Processing Systems - Software for Railway Control and Protection Systems, 2011 